\title{
SERS Detection of Multiple Antimicrobial-Resistant Pathogens using Nanosensors
}

\author{
Hayleigh Kearns, ${ }^{\dagger}$ Royston Goodacre, ${ }^{\ddagger}$ Lauren Jamieson, ${ }^{\dagger}$ Duncan Graham ${ }^{\dagger}$ and Karen Faulds ${ }^{\dagger *}$ \\ † Centre for Molecular Nanometrology, Department of Pure and Applied Chemistry, University of Strathclyde, 99 George \\ Street, Glasgow, G1 1RD, United Kingdom. Email: karen.faulds@strath.ac.uk \\ ¥ The Manchester Institute of Biotechnology, School of Chemistry, University of Manchester, 131 Princess Street, Manchester, \\ M1 7DN, United Kingdom
}

\begin{abstract}
Successful pathogen detection is crucial for public health as the threat of infectious disease is dramatically increasing globally due to bacteria developing resistance to many anti-microbial drugs. The increase in bacterial infections has led to urgent demands for simpler, faster and more reliable detection methods to be developed, so that the most appropriate therapy can be provided. Surface enhanced Raman scattering (SERS) is an analytical technique which has gained a great deal of interest for biosensing due to its sensitivity, selectivity and multiplexing capabilities. A new bionanosensor has been developed for the isolation and detection of multiple bacterial pathogens via magnetic separation and SERS. This novel assay format involves using lectin functionalised magnetic nanoparticles for capture and isolation of bacteria from the sample matrix followed by specifically detecting bacterial pathogens using SERS active nanoparticles functionalised with antibodies which are strain specific. Therefore, the sample is captured using a 'magnetic plug' and interrogated with a laser allowing simple and fast optical detection. Three bacterial pathogens - Escherichia coli, Salmonella typhimurium and methicillin-resistant Staphylococcus aureus - were successfully isolated and detected, with the lowest concentration for each of the strains detected at just $10^{1}$ colony forming units per $\mathrm{mL}(\mathrm{CFU} / \mathrm{mL})$. In addition to single pathogen detection, a mixture of all three bacterial strains were isolated and identified within the same sample matrix using SERS with the triplex detection also being confirmed using principal component analysis. Herein, we demonstrate that this multiplexed bionanosensor is capable of providing rapid and sensitive discrimination of bacterial pathogens both individually, and within a multiplex system, offering opportunities for future point of care devices and advancements in bio-medical applications.
\end{abstract}

Successful pathogen detection is a critical step in ensuring food safety and the health of the general public. ${ }^{1}$ Over the years, there have been many threats to human health from bacterial infections such as Escherichia coli and methicillin-resistant Staphylococcus aureus (MRSA) leading to a surge of ingenious strategies for isolating and detecting microorganisms in connection with food safety, medical diagnostics, water quality, and bioterrorism. ${ }^{2,3}$ Commonly, pathogenic bacteria are detected using conventional methods which rely on lengthy and laborious microbiological, morphological or biochemical identification. Generally these methods fall within three categories; culture-colony counting methods, polymerase chain reaction (PCR) and immunology based techniques such as enzyme-linked immunosorbent assays. ${ }^{1}$ These methods can provide sensitive, qualitative and quantitative data on the microorganisms being analysed, however, they can also be expensive, require specialised equipment with specially trained staff and/or be time consuming as they often require an initial enrichment step. ${ }^{4,5}$ In order to overcome these deficiencies, biosensor technology has grown exponentially in the last 25 years as it has the capability of providing sensitive and reliable results which equate to conventional methods but with much shorter analysis times and the added benefit of analysis being deployed directly on site. ${ }^{4,6,7,8}$

Surface enhanced Raman scattering (SERS) is an ultra-sensitive and selective analytical technique that can be used to detect target molecules attached to or in close-proximity to the surface of noble metal nanoparticles, commonly silver and gold are used. $^{9}$ The enhancements observed are orders of magnitude greater than those reported for conventional Raman scattering, however, the enhancements vary widely depending on analyte and substrate. ${ }^{10,11}$ Additional enhancements, up to $10^{14}$ can be achieved by tuning the laser frequency with an electronic transition within the analyte hence using a chromophore to give rise to the phenomenon known as surface enhanced resonance Raman scattering (SERRS). The main advantage of Raman based techniques are their ability to provide sharp, molecularly specific spectra making it possible to easily discriminate between individual components in a sample mixture, therefore making it an ideal technique for the detection of multiple analytes. ${ }^{12,13}$

To date, there have been many SERS bionanosensors developed which focus on bacterial pathogen detection., ${ }^{2,314-19}$ Zhang et al. used aptamer functionalised gold nanoparticles to detect Salmonella typhimurium and Staphylococcus aureus in pork at concentrations of 15 and $35 \mathrm{CFU} / \mathrm{mL}$ respectively. ${ }^{20}$ Pazos-Perez et al. approached the bacterial detection by designing a 'microorganism optical detection system' consisting of a microfluidic device and micropump which flushed the bacterial sample through the device to a Raman microscope where they analysed the sample using a $785 \mathrm{~nm}$ laser. They reported the detection of multiple bacterial strains at concentrations of $10^{6} \mathrm{CFU} / \mathrm{mL}^{21}$ 
Moreover, there has been an influx in papers recently which use a 'magnetic separation' approach. This involves using magnetic nanoparticles plus a magnet to capture and isolate bacteria from a complex matrix volume, giving rise to increased SERS signal. ${ }^{1,22-26}$ Najafi et al. detected $E$. coli in apple juice at $10^{2}$ $\mathrm{CFU} / \mathrm{mL} ;^{23}$ while Guven et al. reported detection limits of 8 $\mathrm{CFU} / \mathrm{mL}$ in water within $70 \mathrm{~min}{ }^{22}$ Furthermore, Wang et al. reported that $S$. typh and $S$. aureus could be detected simultaneously in spiked spinach and peanut butter at concentrations of $10^{3} \mathrm{CFU} / \mathrm{mL}$, when SERS was combined with a magnetic separation step. ${ }^{1}$ The bionanosensors which have been developed are starting to address the issue of detecting multiple bacterial pathogens simultaneously with 2-3 targets being identified in a sample matrix. They produce reproducible results in comparable times however, they lack the sensitivity, efficiency and ease of our approach. Many of them, require complex separation steps or require dropping the sample onto a surface before analysis, resulting in extra handling steps and increased preparation times. Essentially our approach is a sandwich SERS assay (Figure 1) in which we combine SERS active silver nanoparticles functionalised with bacteria specific recognition molecules (antibodies) with lectin functionalised magnetic nanoparticles enabling the capture and isolation of bacteria through magnetic separation. It is the use of lectin functionalised magnetic nanoparticles, which makes this separation step novel and efficient in capturing and isolating bacteria from a sample matrix.

Lectins are carbohydrate binding proteins that bind specifically to sugars. They have been reported to specifically recognise and bind to carbohydrate constituents expressed on the surface of bacteria. ${ }^{27,28}$ Concanvalin A (Con A) is a type of legume lectin that binds specifically to terminal $\alpha$-D-mannosyl and $\alpha$-D-glucosyl groups present on the surface of all bacteria. ${ }^{29,30}$ In addition, our group has previously reported its use with silver nanoparticles as a molecular imaging agent, investigating carbohydrate-lectin interactions occurring at the surface of mammalian cells using SERS. ${ }^{31}$ Furthermore, we have prior knowledge of combining functionalised silver nanoparticles with silver-coated magnetic nanoparticles in a stable sandwich-type assay for SERS detection of DNA. ${ }^{32}$ Hence, by exploiting our previous knowledge and the fact that lectins bind specifically to sugar moieties on the surface of bacteria, we have in the present study developed a sensitive bionanosensor which is capable of isolating and detecting multiple bacterial pathogens simultaneously. We believe our SERS bionanosensor can provide all the requirements (sensitivity, reproducibility, cost, ease of use and speed of analysis) which are required for a point of care assay and offer opportunities for future advancements in healthcare applications.

\section{Experimental}

Silver nanoparticles and silver coated magnetic nanoparticles were synthesised using methods previously reported by Lee et al. and Kumar et al..$^{33,34}$ The colloidal solutions had an average particle size of $80.9 \mathrm{~nm}$ and $253.3 \mathrm{~nm}$ respectively. The biomoleculenanoparticle conjugates which were used in these studies were prepared using modified methods previously described by Simpson et al. and Donnelly et al. ${ }^{17,32}$ Detailed protocols for the preparation and characterisation of these colloidal suspensions are provided in the experimental section of the ESI.

\section{Bacterial Strains}

Bacterial Strains: Escherichia coli ATCC 25922; Staphylococcus aureus (methicillin resistant) ATCC BAA-1766 and Salmonella typhimurium ATCC 14028 were used in this study. The growth conditions and protocol detailing the preparation of the bacterial slurries are provided in the ESI.

\section{Detection Assay}

Single pathogen detection: For each of the 3 bacterial pathogens the following procedure was carried out - SERS active antibody functionalised silver nanoparticles $(200 \mu \mathrm{L}$; Ab-AgNP) were added together with lectin functionalised silver coated magnetic nanoparticles (200 $\mu \mathrm{L}$; Con A-Ag@MNP) and a specific bacterial strain $\left(50 \mu \mathrm{L}, 10^{4} \mathrm{CFU} / \mathrm{mL}\right)$. Note for the control sample, the bacteria was replaced with d. $\mathrm{H}_{2} \mathrm{O}(50 \mu \mathrm{L})$ only. The sample was mixed thoroughly for $30 \mathrm{~min}$ before being placed in a magnetic rack for a further $30 \mathrm{~min}$ to allow the sample to collect. The clear supernatant was removed and the sample re-suspended in $\mathrm{d} . \mathrm{H}_{2} \mathrm{O}$ $(450 \mu \mathrm{L})$ ready for analysis. The same procedure was followed for the concentration studies except the bacteria concentration was varied from $10^{4}$ to $10^{1} \mathrm{CFU} / \mathrm{mL}$. To confirm concentration dependence, an extended concentration study was conducted for E. coli bacteria only where the assay was performed over the range $10^{7}$ to $10^{1} \mathrm{CFU} / \mathrm{mL}$. It should be noted that each sample was prepared in triplicate.

Multiple pathogen detection: The three sets of antibody functionalised silver nanoparticles $(100 \mu \mathrm{L}$ of each conjugate) were added together with lectin functionalised silver coated magnetic nanoparticles $(200 \mu \mathrm{L})$ plus the three bacterial strains $(50 \mu \mathrm{L}$ of each pathogen; $10^{3} \mathrm{CFU} / \mathrm{mL}$ ). Note for the control sample, the bacteria was replaced with d. $\mathrm{H}_{2} \mathrm{O}(150 \mu \mathrm{L})$ only. The same procedure as described above (for the single pathogen detection) was employed, except the sample was re-suspended in $650 \mu \mathrm{L}$ of d. $\mathrm{H}_{2} \mathrm{O}$.

\section{SERS and Multivariate Analysis}

Raman reporters used were malachite green isothiocyanate (MGITC); 7-dimethylamino-4-methylcoumarin-3-isothiocyanate (DACITC) and 4-(1H-pyrazol-4-yl)-pyridine (PPY) and these were used to detect E. coli, S. typh and MRSA respectively.

The samples were analysed immediately after preparation using a Renishaw InVia Raman microscope. Specifically, the Raman spectra were acquired using a $532 \mathrm{~nm}$ laser excitation with a 20x long working distance objective lens focused into a 96 well plate containing $300 \mu \mathrm{L}$ of the bacteria-NP conjugate solution. The laser power was adjusted each time to $\sim 0.8 \mathrm{~mW}$ and an acquisition time of $1 \mathrm{~s}$ with a static scan being centred at $1300 \mathrm{~cm}$ ${ }^{1}$ was employed. For all Raman spectra, data handling was carried out using GRAMS/AI software. The peak intensities were obtained by scanning 3 replicate samples 5 times and in all plots the error bars represent one standard deviation.

All multivariate statistical analysis was carried out in MATLAB software (The MathWorks, Natick, USA). Prior to conducting principal component analysis (PCA) the data was baseline corrected using asymmetric least-squares (AsLS). ${ }^{35}$ PCA was performed on four different data sets, three consisting of spectra obtained from single pathogen detection experiments 
and one data set obtained for the multiplex detection of the three pathogens. ${ }^{36}$

\section{Results and Discussion}

\section{Characterisation of Biomolecule-NP Conjugates}

The developed bionansosensor is based on a sandwich type SERS assay with the detection strategy provided in Figure 1. Initially, maghemite $\left(\gamma-\mathrm{Fe}_{2} \mathrm{O}_{3}\right)$ magnetic nanoparticles were synthesised using a co-precipitation method, and then coated with a silver shell via a glucose reduction protocol previously described by Donnelly et al. ${ }^{32}$ The silver coated magnetic nanoparticles were functionalised with a heterobifunctional thiol/carboxy PEGylated linker and the legume lectin, Concanvilin A (Con A), via carbodiimide cross-coupling chemistry. ${ }^{17,32,37}$ Conjugating Con A to the surface of silver coated magnetic nanoparticles, in the presence of a magnet allowed for us to develop a unique and efficient way of capturing bacteria from a sample matrix.

Con A functionalised magnetic nanoparticles were prepared using modified methods previously described by Simpson et al. and Donnelly et al. ${ }^{17,32}$ The magnetic nanoparticle conjugates were optimised and characterised using extinction spectroscopy, dynamic light scattering and zeta potential analysis. The data obtained at each stage of the conjugation process (Figure S1) along with a detailed discussion of the results are provided in the ESI. In summary, a shift from 420 to $430 \mathrm{~nm}$ in the localised surface plasmon resonance (LSPR) plus broadening of the peak was observed after the addition of the Con A lectin (Figure S1 (a)). Furthermore, an increase in size and decrease in zeta potential (Figure S1 (c) and (d) respectively) were observed at each stage of the conjugation indicating that there was a change to the surface environment of the nanoparticle and thus confirming the successful attachment of both the PEGylated linker and the Con A lectin to the magnetic nanoparticles.

It was also important to optimise and characterise each step of the conjugation process for the SERS active antibody functionalised silver nanoparticles. Silver nanoparticles were initially synthesised using a modified Lee and Miesel method. ${ }^{33}$ Following this the SERS active conjugates were prepared by functionalising the silver nanoparticles with a Raman reporter, PEGylated linker and antibodies that were bacterial strain specific. Again, carbodiimide cross-linking chemistry was used to immobilise the antibodies onto the nanoparticle surface and the conjugates characterised using the same techniques as those previously employed for the Con A functionalised magnetic nanoparticles. It should be noted that the three sets of antibody conjugates (E. coli, S. typh and MRSA) were optimised and characterised in the same way, with similar trends being observed in the data sets hence, only the data associated with the E. coli antibody is shown in Figure S1 (ESI). A detailed discussion of the characterisation of the SERS active conjugates is provided in the ESI. Briefly, a LSPR shift from 407 to $413 \mathrm{~nm}$ plus broadening and dampening of the peak was observed upon conjugating with the E. coli antibody. In addition, a size increase and decrease in zeta potential (Figure S1 (c and d)) were observed at each stage of the conjugation confirming the successful attachment of the bio-molecule. Moreover, the SERS results showed that with a $1 \mathrm{~s}$ accumulation time and $532 \mathrm{~nm}$ laser an excellent signal could be obtained from the SERS active conjugates (Figure S1 (e)). For further clarification on the successful conjugation and to confirm the stability of the colloidal solutions, gel electrophoresis was performed (Figure S1 (f)). These results along with those observed for the Con A functionalised magnetic nanoparticles suggested that the functionalisation conditions were optimal for use in the next stage of the studies which was developing the assay for single pathogen detection. ${ }^{17}$

\section{Detection Assay}

The detection assay is based on a sandwich-type SERS approach, involving the use of Con A functionalised magnetic nanoparticles and SERS active nanoparticles functionalised with antibodies that are bacterial strain specific. It is important to note that the Raman reporter (highlighted as stars in the schematic in Figure 1) is only present on the bio-recognition nanoparticles and not on the magnetic nanoparticles. Hence, a SERS signal is only observed when the SERS active nanoparticle binds specifically to its bacterial target. As shown in Figure 1 (a-c), a SERS signal is only obtained (green spectrum) when both the bio-recognition nanoparticles and the magnetic nanoparticles bind to the bacterial target. A magnet is then applied and magnetic separation is used to remove the SERS active complex from the matrix volume. In the case where there was no bacterial target present (control sample; red spectrum), the SERS active nanoparticles remain in the bulk solution and are washed away, leaving only the magnetic nanoparticles captured and bound to the magnet. These nanoparticles are non-Raman active, and hence only produce weak background signals. Following the separation step, the SERS active complex and control samples are re-suspended in $\mathrm{d} \cdot \mathrm{H}_{2} \mathrm{O}$ and analysed using a $532 \mathrm{~nm}$ laser. In terms of the multiplexing step (Figure 1d) the procedure described above was followed, except that three separate sets of bio-recognition nanoparticles were prepared each specific for one strain of bacteria only and each with a unique Raman reporter. The Raman reporter produces a molecularly specific spectrum and each reporter contains a characteristic peak which can be used to identify and easily discriminate between the individual components in a sample mixture. ${ }^{38}$ It can be seen in the SERS spectra of Figure 1d, that 3 characteristic peaks have been highlighted $\left({ }^{*}\right)$, each one is unique to a Raman reporter and hence is used to identify which bacterial target is present.

Figure 2 shows the results for each of the three bacterial pathogen tests conducted. Figure 2 (a-c) provides the results for $E$. coli, (d-f) S. typh and (g-i) the results of the MRSA, all of these are single-plex assays. It can be seen in Figure 2 (a) that when both the SERS active biorecognition conjugates and the magnetic conjugates bind to the E. coli bacteria and are pulled from the matrix volume using a magnet, that successful identification of each of the bacteria can be made due to a large SERS signal (green spectrum) being observed. The Raman spectrum is due to the Raman reporter malachite green isothiocyanate (MGITC; chemical structure given in Figure 2 (c)) which was added to the E. coli biorecognition conjugates; therefore it allowed us to confidently confirm the presence of the E. coli bacteria. In terms of the control sample, when no bacteria were added to the matrix volume, it can be seen that only a very weak SERS signal was observed (red spectrum). This weak signal is 
due to residual SERS active biorecognition conjugates not being completely washed away during the separation step. However, this signal is negligible as it is clear there is a large enhancement in the signal observed from the SERS active bacteria complex over the control.

The enhancement in SERS signal from the sample over the control is due to the unique design of this assay. Firstly, the use of Con A functionalised magnetic nanoparticles, allows for multi-valent interactions to take place between the lectin and the sugar moieties on the bacteria surface. ${ }^{30}$ In addition to this, the magnetic core of the nanoparticles enables the use of a magnet in which magnetic separation can be employed to capture and isolate the bacteria-nanoparticle complex from the matrix volume. This allows for sample concentration and any unbound material to be washed away, ultimately reducing the background signal and the chance of false positives. Secondly, the surface of bacteria also provides multiple binding sites for interacting with antibody functionalised nanoparticles. ${ }^{1}$ The biorecognition event, which occurs between SERS active antibody functionalised nanoparticles and antigens present on the surface of bacteria, results in enhanced SERS signals being obtained (as seen in Figure 2 (a)). The multi-valent interaction which occurs enables the nanoparticles to come in-close proximity to one another, resulting in areas of highly localised electromagnetic fields known as SERS hot-spots and these give rise to increased Raman signals. ${ }^{39}$ Therefore, it can be confirmed that the enhancement in SERS signal for the sample over the control was due to the SERS active nanoparticles binding specifically to the bacterial target; with the help of the lectin functionalised magnetic nanoparticles capturing and isolating the bacteria complex from the sample matrix. Thus, the signal enhancement was a result of both conjugates being present and binding to the E. coli bacteria.

When no bacteria were present however, the SERS active biorecognition nanoparticles were unable to bind and therefore could not be pulled down by the magnet during the separation step. Essentially they were washed away with the remaining unbound matrix volume, leaving only the magnetic nanoparticles to be analysed, thus resulting in a very weak Raman signal being obtained. It is evident from Figure 2 (a) and further clarified in Figure $2(b)$ that a signal enhancement of $\sim 10$ times is observed for the E. coli bacteria sample over the control. The characteristic peak at $1616 \mathrm{~cm}^{-1}$ which arises due to aromatic ring stretching within the MGITC molecule, ${ }^{40}$ was used to calculate the peak intensity values. Peak intensities were obtained by scanning 3 replicate samples 5 times and the error bars represent one standard deviation. The total analysis time was $\sim 1 \mathrm{~h}$ from mixing the sample to obtaining the SERS results. Therefore, in comparison to culture based methods where it can take up to 7 days for results to be obtained, a bionanosensor capable of rapidly isolating and simultaneously detecting E. coli bacteria has been demonstrated. We believe however, that this analysis time could be further improved with additional optimisation and automation of the wash steps.

The number of bacterial pathogens which could be detected was extended to $S$. typh, another Gram-negative bacterial strain, and MRSA, a Gram-positive bacterial strain, which were chosen to show the breadth and facile adaptability of the bionanosensor. The results of the single pathogen tests for $S$. typh and MRSA can be observed in Figure $2(\mathrm{~d}-\mathrm{f})$ and $(\mathrm{g}-\mathrm{i})$ respectively.
The Raman reporter DACITC (dimethylamino-4-methylcoumarin-3-isothiocyanate) with a characteristic peak at 535 $\mathrm{cm}^{-1}$ was used to detect $S$. typh, whilst PPY (4-(1H-pyrazol-4-yl)pyridine) with a characteristic peak at $955 \mathrm{~cm}^{-1}$ was used to detect the presence of MRSA. Similar results were observed for these two bacterial strains as those previously seen with the E. coli bacteria, such that intense SERS signals were obtained when the bacteria was present and very weak signals when they were not. In terms of the $S$. typh bacteria, a signal enhancement of 13 times (blue spectrum) was observed for the SERS active bacteria complex over the control sample (red spectrum). Whilst the signal for the MRSA bacteria complex (orange spectrum) over the control sample was $\sim 11$ times greater, thus further demonstrating the level of discrimination which can be achieved using this approach. Moreover, we have demonstrated that this bionanosensor is both speedy and versatile as it is capable of isolating and detecting both Gram-positive and Gramnegative bacterial pathogens. Further, the discrimination which can be achieved between sample and control is undeniable.

\section{Single Pathogen Detection}

For this approach to represent an advancement over current methodologies, it also has to be sensitive as well as fast. Therefore, concentration studies for each of the 3 bacterial pathogens were conducted to determine what the cell concentration limit would be, in terms of discriminating between the SERS active bacteria complex and the control. The assay was performed for each of the pathogens over the bacteria concentration range $10^{4}$ to $10^{1} \mathrm{CFU} / \mathrm{mL}$. Figure 3, shows the SERS spectra obtained at each of the concentrations plus a control spectrum for comparison, for (a) E. coli, (b) S. typh and c) MRSA. Additionally, associated bar charts detailing the SERS peak intensities for each of the bacteria concentrations are also provided. For ease and visual clarification each of the bar chart contains a dashed line, this is used to highlight the peak intensity of the control. Furthermore, an extended concentration study was conducted for E. coli bacteria where the assay was performed over the range $10^{7}$ to $10^{1} \mathrm{CFU} / \mathrm{mL}$. The results from this can be seen in Figure S2 (ESI) but it was obvious that at bacteria concentrations greater than $10^{4} \mathrm{CFU} / \mathrm{mL}$, too many bacteria were present in the sample causing the binding of large numbers of nanoparticles followed by concentration into a small volume by magnetic capture. This resulted in dampening of the SERS signal due to self-absorbance of the Raman scattered light. There was a clear concentration dependence at $10^{4} \mathrm{CFU} / \mathrm{mL}$ and below, and as such the sensitivity was only conducted from $10^{4}$ to $10^{1}$ $\mathrm{CFU} / \mathrm{mL}$. As previously mentioned, the concentration studies produced similar results with the greatest SERS signals being obtained when cell concentrations of $10^{4} \mathrm{CFU} / \mathrm{mL}$ were employed. The weakest SERS responses were observed from the controls therefore, even at low bacteria concentrations of $10^{1}$ $\mathrm{CFU} / \mathrm{mL}$, using this approach we were able to discriminate between the SERS active bacteria complexes and the controls.

In terms of the assay's sensitivity, it is obvious from Figure 3 that detection can confidently be made with cell concentrations between $10^{4}$ and $10^{2} \mathrm{CFU} / \mathrm{mL}$ for all three bacterial pathogens. The signals for the SERS active bacteria complexes at these concentrations are far superior to the controls. The lowest discrimination between the sample and control however, was found to be with a bacteria concentration of $10^{1} \mathrm{CFU} / \mathrm{mL}$. 
For the E. coli bacteria, the SERS active bacteria complex produced a SERS signal twice that of the control, confirming that we were able to discriminate between the two samples and thus able to detect E. coli bacteria at a concentration of $10^{1}$ $\mathrm{CFU} / \mathrm{mL}$. Additionally, it was possible to detect S. typh and MRSA at this concentration also, with signal enhancements of $\sim 1.5$ and 1.7 being observed for the SERS active bacteria complexes over the control samples respectively. It should be noted however, $50 \mathrm{uL}$ of bacteria was used in these assays hence the detection limit is actually lower than $10^{1} \mathrm{CFU} / \mathrm{ml}$, as the number of bacteria present in these samples is less than if $1 \mathrm{~mL}$ of bacteria had been added. Moreover, we have successfully demonstrated that this SERS bionanosensor is not only rapid and versatile but also sensitive in detecting bacterial pathogens. In addition, the bionanosensor is capable of detecting concentrations which are clinically relevant, with current methodologies typically detecting $10^{3} \mathrm{CFU} / \mathrm{mL}$. ${ }^{41,42}$

\section{Multiple Pathogen Detection}

Food contamination is a threat to public health and it can occur at any stage between preparation and consumption; affecting 500,000 people in the UK alone each year. Our bionanosensor is capable of detecting multiple bacterial pathogens simultaneously and in the case of food pathogens where co-infection is plausible we demonstrate the successful detection of both E. coli and S. typh simultaneously (Figure S3). However to demonstrate the diversity and adaptability of this bionanosensor we have chosen to detect food pathogens and MRSA which also has a huge impact on human health. For the multiplex detection assay, the same procedure described previously was followed except three separate sets of bio-recognition nanoparticles were prepared each specific for one strain of bacteria only and each with a unique Raman reporter. Figure 4 (a) shows the SERS spectrum obtained when all three bacterial pathogens ( $E$. coli, S. typh and MRSA) were present in the sample. This multiplex spectrum (black) was compared to the SERS spectra obtained when each of the pathogens were detected individually and a control sample for when no bacteria was present (red).

The DACITC spectrum (blue) represents S. typh; MGITC spectrum (green) represents E. coli; PPY spectrum (orange) represents MRSA. It can be seen in Figure 4 (a) that the black dotted line highlights three peaks at 535, 955 and $1445 \mathrm{~cm}^{-1}$ which are unique to each of the Raman reporters (DACITC, PPY and MGITC) and hence used to identify the presence of each of the bacterial targets (S. typh, MRSA and E. coli) respectively. The peak at $1616 \mathrm{~cm}^{-1}$ used previously to detect E. coli, occurs close to a peak in the MRSA (PPY peak at $1610 \mathrm{~cm}^{-1}$ ) spectrum, therefore to confirm the presence of E. coli the peak at $1445 \mathrm{~cm}^{-1}$ was chosen instead. This bionanosensor provides a 'one pot' detection system for multiple bacterial pathogens but more important than that, it is capable of isolating and discriminating between the bacterial strains being analysed. As the multiplex spectrum is multivariate in nature, it is not always reliable to analyse the spectrum by eye alone, thus multivariate analysis in the form of principal component analysis (PCA) was performed on the multiplex spectrum along with the three single pathogen spectra. Figure 4 (b) shows the PCA scores plot showing the relationship between the multiplex spectra and each of the three single pathogen spectra. PCA is a common method for the analysis of spectroscopic data, ${ }^{7,36}$ and the resulting principal component (PC) scores plot (Figure 4 (b)) clearly illustrates how strong the relationship was between the multiplex spectra and the pathogen spectra with four unique groupings being identified. It can be seen that the single bacterial pathogen samples form the edges of a triangle with the triplex sample clustering in the middle. The black crosses represent the triplex spectra; green circles are E. coli; blue squares are $S$. typh and red diamonds are MRSA. Five scans of 3 replicate samples are shown in the PCA plot and each group is tightly clustered illustrating the excellent reproducibility of the SERS spectra and hence the whole analytical approach including bacterial isolation as well as detection. The SERS spectra from the triplex sample are also clustered tightly together and are clearly separated from the single bacterial samples. Thus, the PCA supports the previous findings in which successful identification and discrimination between the bacterial pathogens in the multiplex can be made. Moreover, it is not only important to be able to discriminate between the bacterial pathogens within the multiplex but it is vital that the bionanosensor is capable of discriminating between the SERS active bacteria complex and the control sample (when no bacteria are added).

The difference in signal intensity between the triplex sample and control is clearly observed in Figure 4 (a), however for further clarification, Figure 4 (c) provides the associated peak intensity values with error bars for the $1616 \mathrm{~cm}^{-1}$ peak. It can be seen that when the bacterial targets were present, an intense SERS spectrum was obtained (black spectrum) and as explained previously this was due to a biorecognition interaction occurring between the nanoparticle conjugates and the three bacterial strains. However, when no bacteria were present, then only a weak Raman signal was observed (red spectrum). The SERS signal for the triplex sample was $\sim 6$ times greater than that observed for the control, demonstrating the effectiveness of this approach. Again, the total analysis time was $\sim 1 \mathrm{~h}$. This is comparable with other bionanosensors which have been developed but where this approach is unprecedented is that it can detect multiple pathogens simultaneously in this time, whereas previous methods are for single pathogen detection only. ${ }^{2,3}$ Furthermore, this bionanosensor is easy to use and can be adapted for use with portable Raman instruments, making it ideal for use with point of care devices. The sensitivity which can be achieved by this bionanosensor is also below the recommended limits currently required for clinical diagnosis (which is $\geq 10^{3}$ $\mathrm{CFU} / \mathrm{mL}$ ). ${ }^{41,42}$ Therefore; it offers significant advancements over existing methods and offers the opportunity for advancements in healthcare applications.

\section{Conclusions}

A SERS bionanosensor which is rapid, sensitive and capable of isolating and detecting multiple bacterial pathogens simultaneously has been demonstrated. This novel approach involves combining a sandwich type assay with magnetic separation in which a magnet is used to capture a SERS active bacteria complex and isolate it from a matrix volume. Using this detection assay, cell concentrations as low as $10^{1} \mathrm{CFU} / \mathrm{mL}$ were readily detected in single pathogen tests for E. coli, S. typh and MRSA. Furthermore, 3 bacterial strains were isolated and detected simultaneously in a multiplex system, with discrimination being made using both PCA and SERS analysis. The sensitivity of this 
bionanosensor has been demonstrated with bacterial concentrations below the requirements for clinical diagnosis being made. This multiplexed bionanosensor is capable of providing fast and sensitive discrimination of bacterial pathogens both individually and within a multiplex system offering opportunities for future advancements in clinical diagnosis and bio-medical applications.

\section{ASSOCIATED CONTENT}

\section{Supporting Information}

Experimental details for NP synthesis, preparation of biomoleculeNP conjugates, bacterial strain and growth conditions plus bacterial sample preparation; experimental and instrumental conditions for characterising biomolecule-NP conjugates, SERS analysis and chemometrics; figures for optimisation and characterisation of NPbiomolecule conjugates plus detailed discussion of the characterisation; figure of the E. coli assay conducted over the bacteria concentration range $10^{7}$ to $10^{1} \mathrm{CFU} / \mathrm{mL}$ and figure demonstrating duplex detection. The Supporting Information is available free of charge on the ACS Publications website.

\section{AUTHOR INFORMATION}

\section{Corresponding Author}

*Professor Karen Faulds; Email: karen.faulds@strath.ac.uk

\section{Author Contributions}

The manuscript was written through contributions of all authors and all authors have given approval to the final version of the manuscript.

\section{ACKNOWLEDGMENT}

$\mathrm{KF}, \mathrm{HK}$ and $\mathrm{RG}$ wish to thank BBSRC for funding; (BB/M018652/1). RG is also grateful to BBSRC for funding Raman spectroscopy (BB/L014823/1).

The research data associated with this paper will become available at the following link: http://dx.doi.org/10.15129/2ab280edd846-464b-b5d4-1222feb0ac77

\section{REFERENCES}

(1) Wang, Y.; Ravindranath, S.; Irudayaraj, J. Anal. Bioanal. Chem., 2011, 399, 1271-1278.

(2) Jarvis, R. M.; Goodacre, R. Chem. Soc. Rev., 2008, 37, 931-936.

(3) Premasiri, W.; Moir, D.; Klempner, M.; Krieger, N.; Jones, G.; Ziegler, L. J. Phys. Chem. B, 2005, 109, 312-320.

(4) Velusamy, V.; Arshak, K.; Korostynska, O.; Oliwa, K.; Adley, C. Biotechnol. Adv., 2010, 28, 232-254.

(5) Leonard, P.; Hearty, S.; Brennan, J.; Dunne, L.; Quinn, J.; Chakraborty, T.; O’Kennedy, R. Enzyme Microb. Technol., 2003, 32, 3. 13.

(6) Lazcka, O.; Del Campo, F. J.; Munoz, F. X. Biosens Bioelectron., 2007, 22, 1205-1217.

(7) Ellis, D. I.; Brewster, V. L.; Dunn, W. B.; Allwood, J. W.; Golovanov, A. P.; Chem. Soc. Rev., 2012, 41, 5706-5727.

(8) Vigneshvar, S.; Sudhakumari, C.; Senthilkumaran, B.; Prakash, H. Front Bioeng Biotechnol., 2016, 4, Article 11, 1-9.

(9) Laing, S.; Gracie, K.; Faulds, K. Chem. Soc. Rev., 2016, 45, 1901 1918.

(10) Oldenburg, S. J.; Westcott, S. L.; Averitt, R. D.; Halas, N. J. J. Chem. Phys., 1999, 111, 4729-4735.
(11) Wang, Y.; Schlücker, S. Analyst, 2013, 138, 2224-2238.

(12) Harper, M. M.; McKeating, K. S.; Faulds, K. Phys. Chem. Chem. Phys., 2013, 15, 5312-5328.

(13) Dougan, J. A.; Faulds, K. Analyst, 2012, 137, 545-554.

(14) Lan, L.; Yao, Y.; Ping, J.; Ying, Y. Biosens Bioelectron., 2017, 91, 504-514.

(15) Li, H.; Chen, Q.; Hassan, M. M.; Chen, X.; Ouyang, Q.; Guo, Z.; Zhao, J. Biosens Bioelectron., 2017, 92, 192-199.

(16) Gracie, K.; Correa, E.; Mabbott, S.; Dougan, J. A.; Graham, D.; Goodacre, R.; Faulds, K. Chem. Sci., 2014, 5, 1030-1040.

(17) Simpson, J.; Craig, D.; Faulds, K.; Graham, D. Nanoscale Horiz., 2016, 1, 60-63.

(18) Ravindranath, S. P.; Wang, Y.; Irudayaraj, J. Sens Actuators B Chem., 2011, 152, 183-190.

(19) Stephen, K. E.; Homrighausen, D.; DePalma, G.; Nakatsu, C. H.; Irudayaraj, J. Analyst, 2012, 137, 4280-4286.

(20) Zhang, H.; Ma, X.; Liu, Y.; Duan, N.; Wu, S.; Wang, Z.; Xu, B. Biosens Bioelectron., 2015, 74, 872-877.

(21) Pazos-Perez, N.; Pazos, E.; Catala, C.; Mir-Simon, B.; Gómez-de Pedro, S.; Sagales, J.; Villanueva, C.; Vila, J.; Soriano, A.; De Abajo, F. J. G.; Alvarez-Puebla, R. A. Sci. Rep., 2016, 6, 29014 -29024.

(22) Guven, B.; Basaran-Akgul, N.; Temur, E.; Tamer, U.; Boyacı, İ. H. Analyst, 2011, 136, 740-748.

(23) Najafi, R.; Mukherjee, S.; Hudson, J.; Sharma, A.; Banerjee, P. Int J Food Microbiol., 2014, 189, 89-97.

(24) Gu, H.; Ho, P.-L.; Tsang, K. W.; Wang, L.; Xu, B. J. Am. Chem. Soc., 2003, 125, 15702-15703.

(25) Gu, H.; Xu, K.; Xu, C.; Xu, B. Chem. Commun., 2006, 0, 941-949. (26) Zhang, L.; Xu, J.; Mi, L.; Gong, H.; Jiang, S.; Yu, Q. Biosens Bioelectron., 2012, 31, 130-136.

(27) Hirabayashi, J. J. Biochem., 2008, 144, 139-147.

(28) Gao, J.; Liu, D.; Wang, Z. Anal. Chem., 2010, 82, 9240-9247.

(29) Shen, Z.; Huang, M.; Xiao, C.; Zhang, Y.; Zeng, X.; Wang, P. G. Anal. Chem., 2007, 79, 2312.

(30) Firon, N.; Ofek, I.; Sharon, N. Infect. Immun., 1984, 43, 10881090.

(31) Craig, D.; McAughtrie, S.; Simpson, J.; McCraw, C.; Faulds, K.; Graham, D. Anal. Chem., 2014, 86, 4775-5782.

(32) Donnelly, T.; Smith, W. E.; Faulds, K.; Graham, D. Chem. Commun., 2014, 50, 12907-12910.

(33) Lee, P.; Meisel, D. J. Phys. Chem., 1982, 86, 3391-3395.

(34) Kumar, G. P.; Rangarajan, N.; Sonia, B.; Deepika, P.; Bull. Mater. Sci., 2011, 34, 207-216.

(35) Eilers, P. H. Anal. Chem., 2004, 76, 404-411.

(36) Kim, D. H.; Jarvis, R. M.; Xu, Y.; Oliver, A. W.; Allwood, J. W.; Hampson, L.; Hampson, I. N.; Goodacre, R. Analyst, 2010, 135, 1235. 1244 .

(37) Zheng, M.; Davidson, F.; Huang, X. J. Am. Chem. Soc., 2003, 125, 7790-7791.

(38) Faulds, K.; Jarvis, R.; Smith, W. E.; Graham, D.; Goodacre, R. Analyst, 2008, 133, 1505-1512.

(39) Kleinman, S. L.; Frontiera, R. R.; Henry, A.-I.; Dieringer, J. A.; Van Duyne, R. P. Phys. Chem. Chem. Phys., 2013, 15, 21-36.

(40) Smith, E.; Dent, G.; Wiley, J.: Modern Raman spectroscopy: a practical approach; Wiley Online Library, Chichester, 2005.

(41) Goldman, E.; Green, L. H.: Practical handbook of microbiology; CRC Press, London, 2015.

(42) Balows, A.; William Jr, J.; Ohashi, M.; Turano, A.: Laboratory diagnosis of infectious diseases: principles and practice; Springer Science \& Business Media, New York, 2012. 
a)
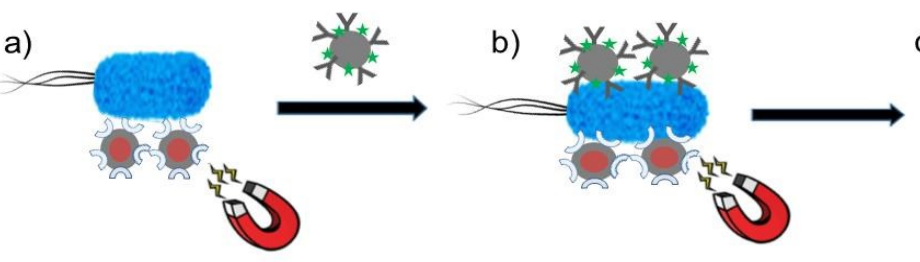

c)

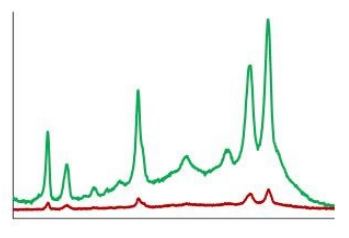

d) Multiplexing Step
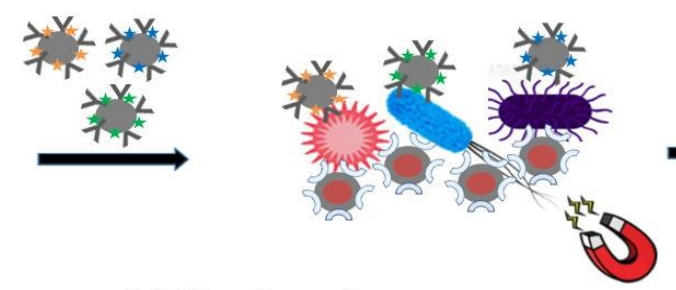

3 SERS active conjugates:
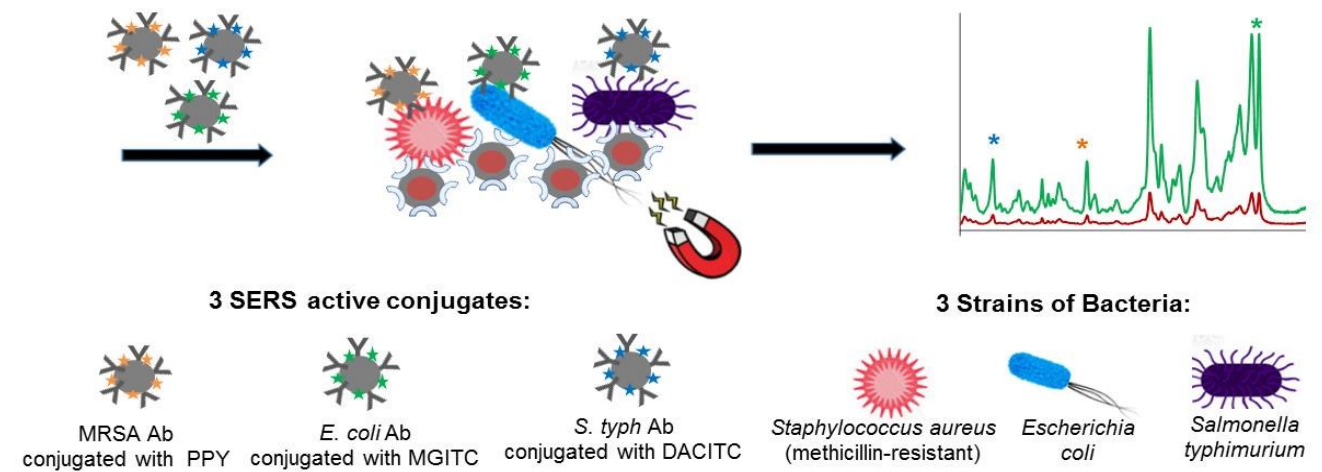

Figure 1. Schematic illustrating the single-plex and multiplex detection assay. Assay format: a) lectin (Con A) functionalised silver coated magnetic nanoparticles ( $\mathrm{Ag} @ \mathrm{MNPs})$ will bind to bacteria and the presence of the magnet will allow for magnetic separation of the bacteria from the sample matrix b) SERS active silver nanoparticles (AgNPs) functionalised with a biorecognition molecule (antibody; Ab) and a unique SERS reporter are added. The mixture is shaken for $30 \mathrm{~min}$ before applying a magnet for a further $30 \mathrm{~min}$ and allowing the sample to collect. Any unbound matrix is gently removed and the sample subsequently re-suspended in $\mathrm{d} \cdot \mathrm{H}_{2} \mathrm{O}$ c) The sample is then interrogated with a $532 \mathrm{~nm}$ laser beam and SERS signal obtained (green spectrum). When no target is present the functionalised AgNPs will be washed away, thus they will not bind to bacteria so a minimum SERS signal obtained (red spectrum). d) Multiplexing step: 3x AgNP conjugates each functionalised with a different Raman reporter and an antibody (which is specific for a bacterial pathogen) are added together with 3 bacterial pathogens and Con A (which binds to all three bacteria) functionalised Ag@MNPs. In the same way as the single-plex systems magnetic separation allows for the samples to be concentrated and analysed via a $532 \mathrm{~nm}$ laser. A SERS spectrum is obtained which contains characteristic peaks from the three Raman reporters and thus can be used to confirm the targets are present.
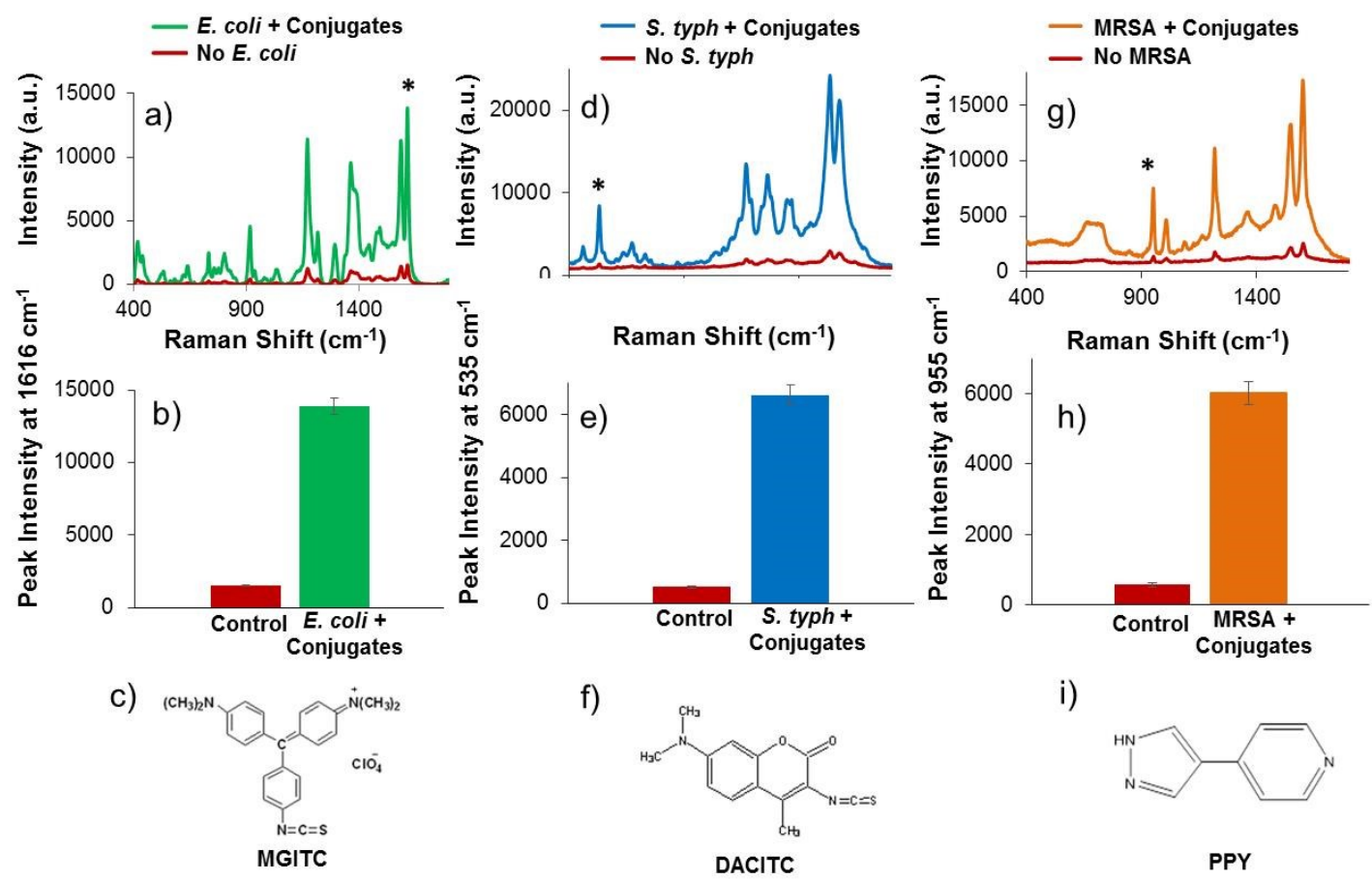

f)

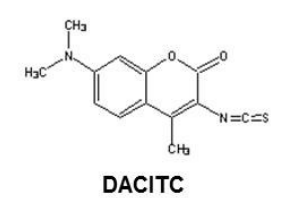

i)

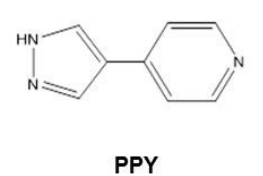

Figure 2. SERS spectra obtained from single pathogen detection using the SERS assay. a) SERS spectrum of MGITC observed when detecting E. coli (green) and the control spectrum representing when no bacteria was present (red); b) SERS peak intensities at $1616 \mathrm{~cm}^{-1}$ for assay and control when detecting E. coli; c) Chemical structure for the Raman reporter, MGITC; d) SERS spectrum of DACITC observed when detecting $S$. typh (blue) and the control spectrum (red); e) SERS peak intensities at $535 \mathrm{~cm}^{-1}$ for assay and control when detecting $S$. typh; f) Chemical structure for DACITC; g) SERS spectrum of PPY observed when detecting MRSA (orange) and the control spectrum (red); h) SERS peak intensities at $955 \mathrm{~cm}^{-1}$ for assay and control when detecting MRSA; i) Chemical structure for PPY. The bacteria concentration used was $10^{4} \mathrm{CFU} / \mathrm{mL}$. SERS spectra were recorded using a $532 \mathrm{~nm}$ laser excitation with an accumulation time of $1 \mathrm{~s}$. Peak 
intensities were obtained by scanning 3 replicate samples 5 times and the error bars represent one standard deviation. The (*) highlights a characteristic peak which is used to identify the Raman reporter hence the bacterial pathogen present but it also used to calculate the peak intensities throughout.

a)
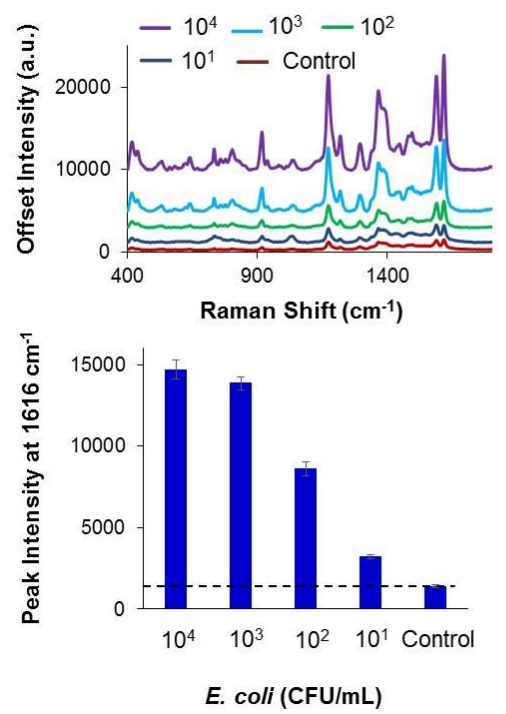

b)
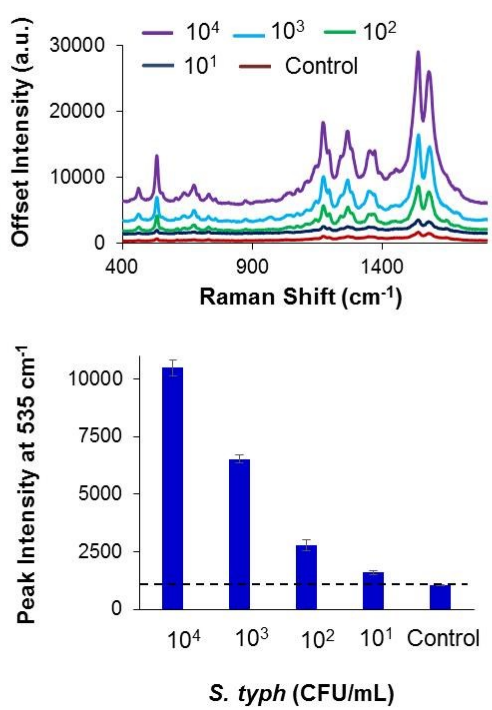

c)

MRSA
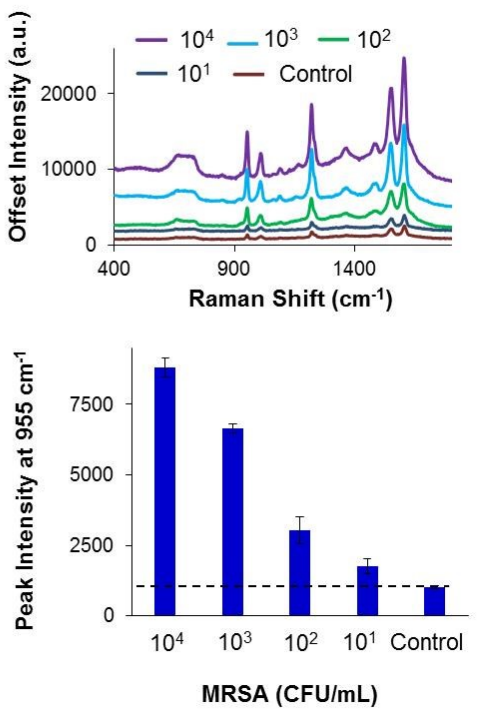

Figure 3. The assay was performed for each of the pathogens over the bacteria concentration range $10^{4}$ to $10^{1} \mathrm{CFU} / \mathrm{mL}$. Comparison SERS spectra showing changes in signal for a) MGITC detecting E. coli, b) DACITC detecting S. typh and c) PPY detecting MRSA. The associated bar charts show the SERS peak intensities at each of the bacteria concentrations and these are compared to a control sample (no bacteria present). The characteristic peaks 1616,535 and $955 \mathrm{~cm}^{-1}$ were used to calculate the peak intensities for $E$. coli, S. typh and MRSA respectively. SERS spectra were recorded using a $532 \mathrm{~nm}$ laser excitation with an accumulation time of $1 \mathrm{~s}$. Peak intensities were obtained by scanning 3 replicate samples 5 times and the error bars represent one standard deviation. The dashed line gives visual clarification of the SERS peak intensity of the control.
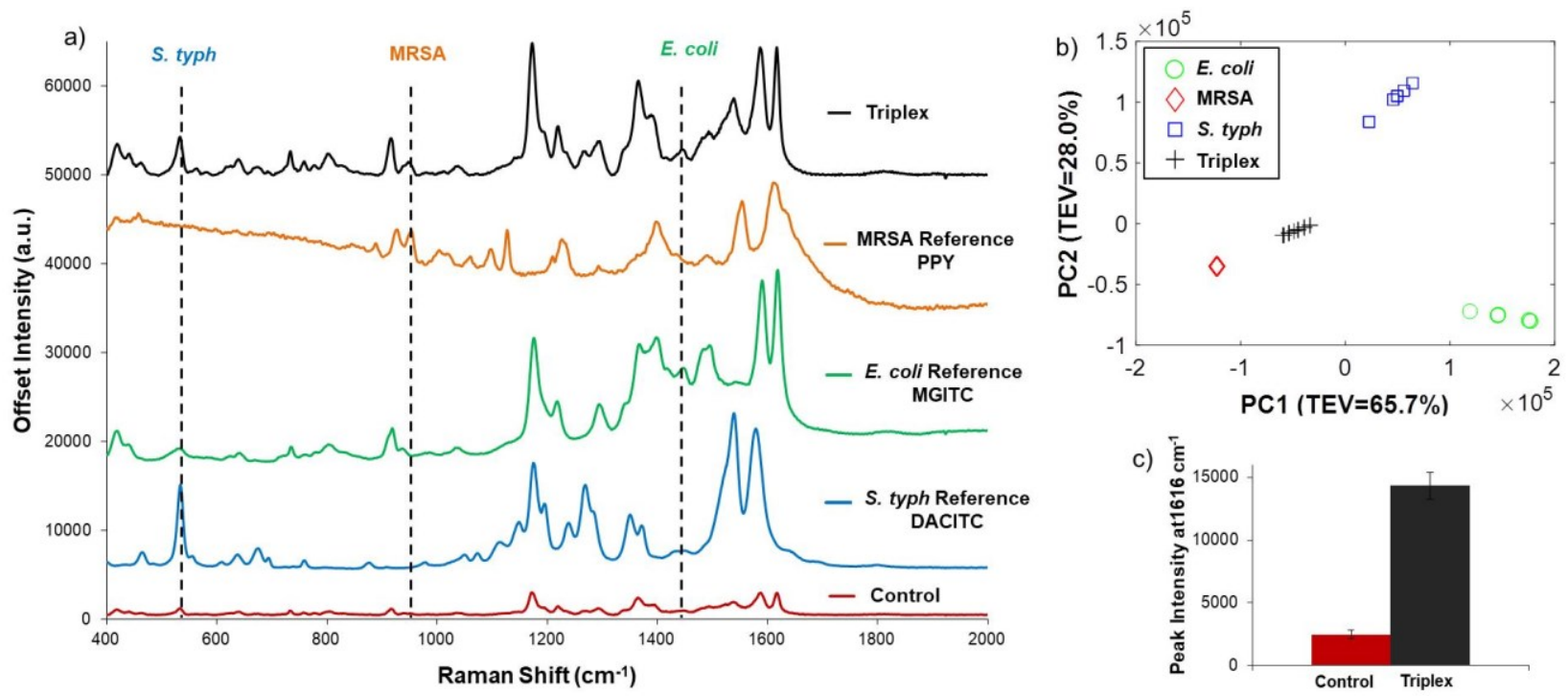

Figure 4. SERS spectra obtained from multiple pathogen detection using the SERS assay. a) Stacked SERS spectra showing the raw spectra obtained from the detection of all three bacterial pathogens simultaneously using the detection assay and a control sample for comparison when no bacteria was present plus the SERS spectra obtained from the detection of each pathogen separately. Control spectrum is red, DACITC spectrum (blue) represents $S$. typh; MGITC spectrum (green) represents E. coli; PPY spectrum (orange) represents MRSA and the black spectrum is the multiplex containing all three bacterial pathogens. The black dotted lines show peaks that are unique to each Raman reporter and hence used to identify the presence of the bacterial targets. The bacteria concentration used was $10^{3} \mathrm{CFU} / \mathrm{mL}$. b) PCA scores plot showing the relationship between the multiplex spectra and each of the three single pathogen spectra. The black crosses represent the triplex spectra; green circles are E. coli; blue squares are $S$. typh and red diamonds are MRSA. c) Comparative peak intensities at $1616 \mathrm{~cm}^{-1}$ for assay and control. SERS spectra were recorded using a $532 \mathrm{~nm}$ laser excitation with an accumulation time of $1 \mathrm{~s}$. Peak intensities were obtained by scanning 3 replicate samples 5 times and the error bars represent one standard deviation. TEV $=$ total explained variance. 
For TOC Only:

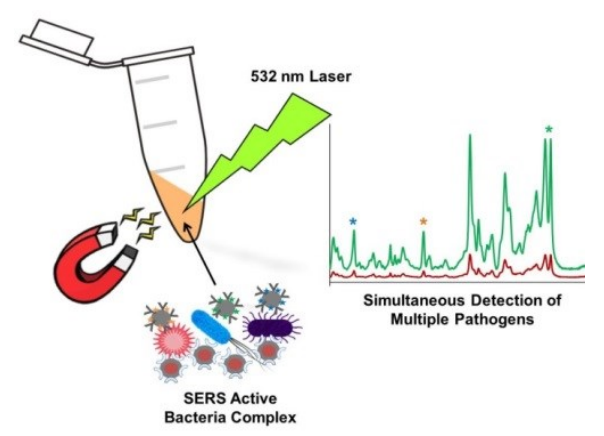

